\title{
Android Application Suite for Student Tracking System
}

\author{
Kunal Vilas Chaudhari \\ Department of Computer \\ Engineering, \\ Ramrao Adik Institute of \\ Technology, Nerul, \\ Navi-Mumbai.
}

\begin{abstract}
In today's world of E-learning, webinars and video conferencing, we still rely on paper sheets and manual human workload for marking and managing student attendance, results. Also this handwritten is to be converted into digital format for one or the other reasons like result analysis or making defaulters list etc. Irrespective of the subject, the higher authority of the college has to analyze this data for all students. In this traditional method which is commonly used in all colleges, interaction of this student data (results, attendance) with the guardian of student have remarkable issues. So due to this it is necessary to develop an automated system for student tracking. In this paper, I describe a proposed android application suite for student tracking system. This application suite will bridge the gap between the students, teachers, higher authorities of college and the guardians.
\end{abstract}

\section{Keywords}

Android, Attendance, Results, Student Tracking System, Guardians.

\section{INTRODUCTION}

Traditional method of marking student attendance is very simple yet time consuming. The teacher passes a sheet of paper during the lecture than the students sign it and pass it back to the teacher. In the present system all work is done on paper [1]. This process is time consuming and is prone to human errors. In this paper I am proposing an application suite out of which an application will be installed on the android device of student and the teacher each. Also the other modules will be installed on the android device of the guardians and higher authority of the college to analyze the result and attendance.

\section{EXISTING SYSTEM}

Let us take a look at the existing system of student tracking according to various participants of system like teachers, students, higher authority, and guardians.

\subsection{Teachers}

Teachers will be most beneficial by this application suite. Traditionally the teacher has to call roll numbers of students or pass a sheet of paper to mark the student attendance. Also at end of the term or during the need to analyze this data, teachers need to feed this data into the computer manually and generate reports like defaulters list. Also the teachers have to submit a detailed report to the higher authority which again requires manual interaction. The teacher if wants to schedule an extra lecture or give students the list of assignments, she or he has to follow the protocols to put the details on the notice board.

\subsection{Students}

Students are also the main crucial part in this system. Although if anything fails in the traditional system the students face problems. Traditionally the student has to answer roll calls or sign on a sheet of paper supplied by the respective subject teacher. After then they also have to wait for the defaulters list to be displayed which will be generated after teacher completes the generation of report manually at the time specified by the higher authority or teacher. The traditional way for a student to check his updated attendance records is to contact the respective teacher and ask her.

Also if the students want to check the updated time tables, extra lectures or meetings, results, assignments list, tutorials the traditional method will be to follow the notice board or the respective teacher.

\subsection{Higher authority}

The higher authority of the college may include various people like office administrators, principal, dean etc. These are the people who are responsible for the analysis of the results and attendance of the students. According to different protocols and rules, the higher authority calls for the reports from the teachers for tracking student information like results, attendance, and extra-curricular activities. This very difficult for them to analyze the data as well as take suitable decisions on different scenarios. In this aspect too it is tedious job for higher authorities to inform teachers, students, regarding meetings, instructions or protocols to be followed.

\subsection{Guardians}

During all the process by traditional method, the guardians are most deprived of facilities and not taken care of. The guardians are almost unaware of their children's activities in college as well as different aspects like attendance, results etc. In the traditional method the guardian has to call or visit the college to track their child's attendance or result. Although there is no guarantee that the records provided to them may be updated because traditional method is very time consuming.

Now we have all studied a broad look of traditional approach followed by different participants to track the students. This whole system is very unreliable and time consuming. In this paper I will propose an android application suite which will be a combination for all these participants. There will be different applications in this suite. 


\subsection{Disadvantages of existing system}

- There are many disadvantages in the conventional system. This system is less organized as well as has less flexibility.

- The system uses lot of paper which is wasted at the end of the day

- It is time consuming as well as less user friendly.

- The system is less integrated as it does not hold together the different participants in system such as students, teachers, etc.

- Also it has less availability as students cannot access their attendance easily.

\section{PROPOSED SYSTEM}

Basically the proposed android suite will consists of series of android applications based upon the different participants. The different components will be as follows

1. Student application.

2. Teacher application.

3. High command application.

4. Guardian application.

These are the broadly classified components of the suite.

\subsection{Student application}

The student application will be designed to provide easy and automated attendance to the teaching staff of the organization. The student application will consist of two modes as follows:-

\subsubsection{Lecture Mode}

In the lecture mode, the student's android device will scan for the teacher's application (Wi-Fi network created by teacher's application) and send data to the staff android device for his/her attendance. The student's android device id will be registered in the database earlier and then the teacher's application will scan all the students and mark their attendance automatically. This mode does not require internet connectivity on the student's android device.

\subsubsection{College Mode}

If this mode is selected by the student then the internet connectivity is mandatory on android device used by him/her. With the help of internet, the students can get alerted regarding different meetings, extra lectures, assignments, time tables, his attendance, defaulter's list, etc. This data will be gathered from the database which will be managed by the teacher's application. The students will also have a chat section to get in touch with their classmates. They also will be able to manage their assignments and analyze their attendance.

\subsection{Teacher Application}

The teacher application will be most crucial part in this application suite. As we have seen in student application, this application will also have two modes.

\subsubsection{Lecture Mode}

In this mode, the teacher's android device will create a Wi-Fi network and wait for the student's device to accept the connection. The teacher's application will be aware of the device id of each student respectively. The application if connects to the teacher's Wi-Fi network then the respective student is marked present. For avoiding false proxies, the teacher's application network will be available only for specific time duration. Only for specific time duration, the student has to start lecture mode. If an android device or application is not available with the student then the student has to use biometrics system (fingerprint) on the teacher's application. If the teacher thinks there has been a proxy attendance, then he/she can manually mark the attendance in the list developed. The lecture for which the attendance to be marked will be automatically selected on the basis of teacher's allotted schedule.

\subsubsection{College Mode}

In this mode, the internet connection is required on android device. The teacher's application will be updated regarding the student's device id and their personal information. In this application, the teacher can manage his/her allotment schedule of lectures and also if it has been delivered by some higher authority like principal then it will be reflected directly from the college database online. The teacher can analyze the attendance or sort out queries and problems if any and submit the final list to the higher authority for analysis.

The teacher can also update any time tables, meetings, assignments, extra lectures for students in the database which will be further reflected to student's application. Some colleges have biometrics used for staff attendance, so it will be collected from database of college to map the data updated from teacher's application regarding student report and defaulter's list etc.

\subsection{High command application}

This application will be specific for the higher authorities in college like principal, dean office staff, etc. This application will be the most sophisticated application of the entire application suite. The higher authority can update any meeting timings, analyze the attendance of teachers, students, and other staff.

Also the higher authorities can keep in touch with the students and the teachers directly. They can update any notices or arrange/broadcast any important notification to the students or their teachers or the guardians. This application will need internet connectivity on the android device for operating.

\subsection{Guardian application}

This is the important part of the application suite. This application will act as a bridge between the college and the guardians. The guardians will be updated regarding the students records like attendance, results, college schedules, notifications. This application will help to reduce the time to recover the student's record which is formally too large in the traditional method.

These are the different application features explained and their sophistication in brief. The further sections will explain the actual working of the system if implemented. 


\section{FLOW DIAGRAM}

The flow diagram of the application suite will follow different paths and according to different perspectives. The flow diagram will give the outlook of the application suite and working of the application suite being integrated. The integration of this application suite is very important with respect to all the perspectives.

\section{ADVANTAGES}

This proposed system will be very beneficial to reduce the manual human work load. The advantages of the android application suite are listed below.

$>$ Time efficient.

$>$ High Integration and availability.

$>$ Less Paper work.

$>$ Easy to implement.

$>$ Automated system.

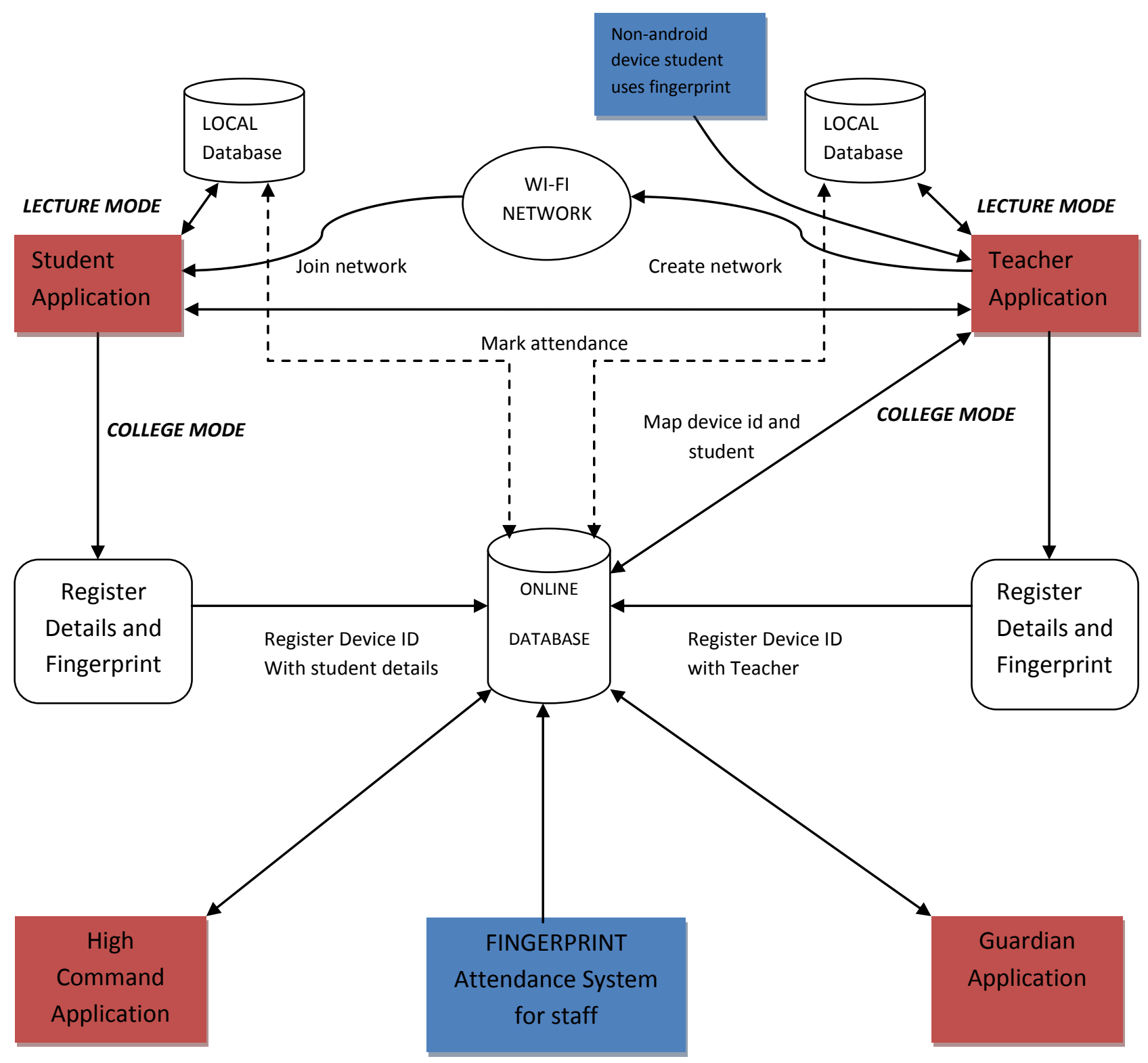

Figure 1: Flow diagram of android application suite. 


\section{CONCLUSION}

In this paper I have proposed an extremely useful yet simple android application suite for student tracking. This application suite will be useful for all the participants allied to the student tracking. This suite enhances the time factor efficiency as well as develops an ease to integrate all the factors easily. We can easily implement this suite in android as it is rapidly growing technology and is easy to use. This paper also discusses the essential basic work flow of the suite which when followed will be easy to implement the same.

\section{FUTURE WORK}

As this paper discusses the proposed system, there is lot to be yet discovered in future. The application suite can also be developed for laboratories and library using similar approach. As well as we can also implement this system on cloud platform. Advanced security features can be incorporated to enhance this system like network security. Also, the false proxy attendance can be handled in different advanced methods.

\section{REFERENCES}

[1] K. Akhila, B. Prathyusha, M. PavanKumar, M. Amrutha. "A Novel Approach of Mobile Based Student Attendance Tracking System Using Android Application". International Journal of Engineering Research \& Technology (IJERT) Vol. 2 Issue 4, April 2013 ISSN: 2278-0181.

[2] Akshay A. Kumbhar, Kunal S. Wanjar, Darshit H. Trivedi, Anay U. Khairatkar and Deepak Sharma. "Automated Attendance Monitoring System using Android Platform". International Journal of Current Engineering and Technology, E-ISSN 2277 - 4106, PISSN $2347-5161$.

[3] Vijay Kakani, "Implementation of Reliable Attendance System using Android and Near Field Communication Technology", Global Journal of Design, Engineering and Technology, Vol.3 (2):5-10.

[4] Namrata N. Shahade, Priya A. Kawade, Satish L.Thombare, "Student Attendance Tracker System in Android", International Journal for Engineering applications and technology. ISSN: 2321-8134. 